\title{
The homotopy Lie algebra of a complex hyperplane arrangement is not necessarily finitely presented.
}

\author{
Jan-Erik Roos \\ Department of Mathematics \\ Stockholm University \\ SE-106 91 Stockholm, SWEDEN \\ e-mail: jeroos@math.su.se
}

October 3, 2006

\begin{abstract}
.
We present a theory that produces several examples where the homotopy Lie algebra of a complex hyperplane arrangement is not finitely presented. This answers a question of Denham and Suciu.

Mathematics Subject Classification (2000): Primary 16E05, 52C35; Secondary 16S37, $55 \mathrm{P} 62$

Keywords. Hyperplane arrangement, homotopy Lie algebra, Yoneda Ext-algebra

\section{Introduction}

Let $\mathcal{A}=\{H\}$ be a set of complex hyperplanes in $\mathbf{C}^{n}$, i.e. a complex hyperplane arrangement in $\mathbf{C}^{n}$ and let $X$ be the complement of their union in $\mathbf{C}^{n}$ :

$$
X=\mathbf{C}^{n} \backslash \bigcup_{H \in \mathcal{A}} H
$$

The cohomology of $X$ is called the Orlik-Solomon algebra and the Yoneda Ext-algebra of $H^{*}(X)$ is a Hopf algebra which is the enveloping algebra of a graded Lie algebra, which is called the homotopy Lie algebra of the arrangement $\mathcal{A}$. In this paper we calculate explicitly this Lie algebra in several cases and in particular I show by explicit examples that this Lie algebra is not necessarily finitely presented and not even finitely generated (which solves an open problem from [De-Su], Question 1.7 p. 321).
\end{abstract}

\section{An explicit example}

It is useful to begin with an explicit example. Let $\mathcal{A}=\{x, y, z, x+y, x+z, y+z\}$ be the well-known complex hyperplane arrangement, which is the smallest formal arrangement 
whose Orlik-Solomon algebra is nonquadratic (cf. [Sh-Yu], p. 487, Example 5.1). We know that the Orlik-Solomon algebra of $\mathcal{A}$ is the quotient of the exterior algebra in 6 variables $e_{1}, e_{2}, e_{3}, e_{4}, e_{5}, e_{6}$ by the twosided ideal generated by the four elements:

$$
\left(e_{2}-e_{6}\right)\left(e_{3}-e_{6}\right), \quad\left(e_{1}-e_{3}\right)\left(e_{3}-e_{5}\right), \quad\left(e_{1}-e_{4}\right)\left(e_{2}-e_{4}\right), \quad\left(e_{3}-e_{4}\right)\left(e_{4}-e_{6}\right)\left(e_{5}-e_{6}\right)
$$

Thus if we introduce new variables $x_{1}, x_{2}, x_{3}, x_{4} \cdot x_{5}, z$ by $x_{i}=e_{i}-e_{6}$ for $1 \leq i \leq 5$ and $z=e_{6}$ our Orlik-Solomon algebra can be written (we use that $e_{1}-e_{4}=\left(e_{1}-e_{6}\right)-\left(e_{4}-e_{6}\right)=$ $x_{1}-x_{4}$ etc.) as a quotient of an exterior algebra:

$$
O S_{\mathcal{A}}=\frac{E\left(x_{1}, x_{2}, x_{3}, x_{4}, x_{5}, z\right)}{\left(x_{2} x_{3},\left(x_{1}-x_{3}\right)\left(x_{3}-x_{5}\right),\left(x_{1}-x_{4}\right)\left(x_{2}-x_{4}\right),\left(x_{3}-x_{4}\right) x_{4} x_{5}\right)}
$$

where $z$ does not occur among the relations. Therefore the Orlik-Solomon algebra decomposes into a tensor product of algebras (all algebras are considered over a field $k$ of characteristic 0):

$$
O S_{\mathcal{A}}=\frac{E\left(x_{1}, x_{2}, x_{3}, x_{4}, x_{5}\right)}{\left(x_{2} x_{3}, x_{1} x_{3}-x_{1} x_{5}+x_{3} x_{5}, x_{1} x_{2}-x_{1} x_{4}+x_{2} x_{4}, x_{3} x_{4} x_{5}\right)} \otimes_{k} E(z)
$$

where $E(z)$ is the exterior algebra in one variable and where we have used that $x_{i}^{2}=0$. Thus the Yoneda Ext-algebra of the Orlik-Solomon algebra is the tensor product of the Ext-algebra $\operatorname{Ext}_{R}^{*}(k, k)$ of

$$
R=\frac{E\left(x_{1}, x_{2}, x_{3}, x_{4}, x_{5}\right)}{\left(x_{2} x_{3}, x_{1} x_{3}-x_{1} x_{5}+x_{3} x_{5}, x_{1} x_{2}-x_{1} x_{4}+x_{2} x_{4}, x_{3} x_{4} x_{5}\right)}
$$

and the Ext-algebra $\operatorname{Ext}_{E(z)}^{*}(k, k)=k[Z]$ where the last algebra is the polynomial algebra in one variable $Z$, dual to $z$. The last algebra is "innocent" and it therefore follows that the Yoneda Ext-algebra of the Orlik-Solomon algebra is finitely presented if and only if $\operatorname{Ext}_{R}^{*}(k, k)$ is so, where $R$ is given by (1.1). But the following automorphism of $R$

$$
x_{1} \rightarrow x_{1}+x_{3}, x_{2} \rightarrow-x_{2}+x_{3}, x_{3} \rightarrow x_{3}, x_{4} \rightarrow-x_{2}+x_{3}+x_{4}, x_{5} \rightarrow x_{5}+x_{3}
$$

transforms $R$ into the isomorphic algebra

$$
\frac{E\left(x_{1}, x_{2}, x_{3}, x_{4}, x_{5}\right)}{\left(x_{2} x_{3}, x_{1} x_{5},\left(x_{1}+x_{2}\right) x_{4}, x_{3} x_{4} x_{5}\right)}
$$

which we will still denote by $R$. But this algebra $\left(^{* *}\right)$ can now be easily analyzed: It is the "trivial extension" of a Koszul algebra

$$
S=\frac{E\left(x_{1}, x_{2}, x_{3}, x_{5}\right)}{\left(x_{2} x_{3}, x_{1} x_{5}\right)}
$$


by the following cyclic module $M$ over $S$ :

$$
M=\frac{S}{\left(x_{1}+x_{2}, x_{3} x_{5}\right)}
$$

Recall that the trivial extension of any $\operatorname{ring} \Lambda$ by any two-sided $\Lambda$-module $N$ is denoted by $\Lambda \propto N$ and consists of the pairs $(\lambda, n)$ with $\lambda \in \Lambda$ and $n \in N$ with pairwise addition and multiplication

$$
(\lambda, n) \cdot\left(\lambda^{\prime}, n^{\prime}\right)=\left(\lambda \cdot \lambda^{\prime}, \lambda n^{\prime}+n \lambda^{\prime}\right)
$$

The Ext-algebra of $R=S \propto M$ can now be analyzed ( cf. e.g. [Lö2,Theorem 3, p. 310-311]): We have a split extension of Hopf algebras

$$
k \rightarrow T\left(s^{-1} \operatorname{Ext}_{S}^{*}(M, k)\right) \rightarrow \operatorname{Ext}_{R}^{*}(k, k) \rightarrow \operatorname{Ext}_{S}^{*}(k, k) \rightarrow k
$$

Here $S$ is the Koszul algebra (1.3) and

$$
\operatorname{Ext}_{S}^{*}(k, k)=k<X_{1}, X_{5}>\otimes_{k} k<X_{2}, X_{3}>
$$

is the tensor product of two free algebras in the dual variables $X_{1}, X_{5}$ and $X_{2}, X_{3}$ respectively, and therefore it has global dimension 2. Furthermore

$$
s^{-1} \operatorname{Ext}_{S}^{*}(M, k)=\operatorname{Ext}_{S}^{*-1}(M, k)
$$

and $T\left(s^{-1} \operatorname{Ext}_{S}^{*}(M, k)\right)$ is the free algebra on the graded vector space (for the ${ }^{*}$-grading in Ext) $s^{-1} \operatorname{Ext}_{S}^{*}(M, k)$ and has global dimension 1 . The spectral sequence of extensions of Hopf algebras (1.4) [Ro2]

$$
E_{p, q}^{2}=\operatorname{Tor}_{p}^{E x t_{S}^{*}(k, k)}\left(k, \operatorname{Tor}_{q}^{T\left(s^{-1} \operatorname{Ext}_{S}^{*}(M, k)\right)}(k, k) \Rightarrow \operatorname{Tor}_{n}^{E x t_{R}^{*}(k, k)}(k, k)\left(=H_{n}\right)\right.
$$

shows immediately that $\operatorname{Ext}_{R}^{*}(k, k)$ har global dimension 3. Furthermore (1.5) degenerates into an long exact sequence:

$$
0 \rightarrow E_{2,1}^{2} \rightarrow H_{3} \rightarrow E_{3,0}^{2} \rightarrow E_{1,1}^{2} \rightarrow H_{2} \rightarrow E_{2,0}^{2} \rightarrow E_{0,1}^{2} \rightarrow H_{1} \rightarrow E_{1,0}^{2} \rightarrow 0
$$

where the natural maps $H_{i} \longrightarrow E_{i, 0}^{2}$ are onto. Indeed, the natural ring projection map $S \propto M \longrightarrow S$ is split by the natural ring inclusion $S \longrightarrow S \propto M$ and this leads to a splitting on the Ext-algebra level. Thus we have exact sequences

$$
0 \longrightarrow E_{i-1,1}^{2} \longrightarrow H_{i} \longrightarrow E_{i, 0}^{2} \longrightarrow 0
$$


Now $H_{1}$ measures the minimal number of generators of the Ext-algebra $E x t_{R}^{*}(k, k)$ and therefore $H_{1}$ is finite-dimensional if and only if the Ext-algebra $E x t_{R}^{*}(k, k)$ is finitely generated. Similarly $\mathrm{H}_{2}$ measures the minimal number of relations in a minimal presentation of $\operatorname{Ext}_{R}^{*}(k, k)$ and $\mathrm{H}_{3}$ measures the minimal number of relations between the relations (cf. Lemaire [Lem]). Since the $E_{i, 0}^{2}$ are all finite-dimensional we are led to the study of

$$
E_{i, 1}^{2}=\operatorname{Tor}_{i}^{E x t_{S}^{*}(k, k)}\left(k, s^{-1} \operatorname{Ext}_{S}^{*}(M, k)\right), \text { for } i \geq 1
$$

where the left $\operatorname{Ext}_{S}^{*}(k, k)$-module structure of $s^{-1} \operatorname{Ext}_{S}^{*}(M, k)$ is given by the Yoneda product (cf. again Theorem 3, p. 310-311 of Löfwall [Lö2]). Note that underlying our spectral sequence is the Hochschild-Serre spectral sequence and that we are in the skewcommutative setting, whereas [Lö2] is in the commutative case, but similar (easier) proofs work here in our case. Thus to show that $\operatorname{Ext}_{R}^{*}(k, k)$ is not finitely generated we have to show that $s^{-1} \operatorname{Ext}_{S}^{*}(M, k)$ needs an infinite number of generators as an $\operatorname{Ext}_{S}^{*}(k, k)$-module, i.e. we have to study the $S$-resolutions of $M=S /\left(x_{1}+x_{2}, x_{3} x_{5}\right)$. We also need the extra grading on $R$ and $S$ so that we should indeed write $R=S \propto s^{-1} M$. Now we denote the $S$-ideal $\left(x_{1}+x_{2}, x_{3} x_{5}\right)$ by $I$ so that $M=S / I$. First we observe that if we apply the functor $\operatorname{Ext}_{S}^{*}(., k)$ to the exact sequence of graded left $S$-modules

$$
0 \longrightarrow I \longrightarrow S \longrightarrow S / I \longrightarrow 0
$$

we obtain the isomorphisms of left $\operatorname{Ext}_{S}^{*}(k, k)$-modules:

$$
\operatorname{Ext}_{S}^{*-1, t}(I, k) \stackrel{\sim}{\longrightarrow} \operatorname{Ext}_{S}^{*, t}(S / I, k), \text { for } * \geq 1
$$

where we also have inserted the inner grading $t$ that comes from the fact that (1.8) is an exact sequence of graded modules. Note that $S$ is a Koszul algebra, so that only the $\operatorname{Ext}_{S}^{i, i}(k, k)$ are different from zero and we still denote them by $\operatorname{Ext}_{S}^{i}(k, k)$. Next we note that the two ideals $I_{1}=\left(x_{1}+x_{2}\right)$ and $I_{2}=\left(x_{3} x_{5}\right)$ in $S$ have zero intersection. Therefore $I=I_{1} \oplus I_{2}$ and the $\operatorname{Ext}_{S}^{*}(k, k)$-module to the left in (1.9) decomposes into a direct sum of $\operatorname{Ext}_{S}^{*}(k, k)$-modules:

$$
\operatorname{Ext}_{S}^{*-1, t}\left(\left(x_{1}+x_{2}\right), k\right) \oplus \operatorname{Ext}_{S}^{*-1, t}\left(\left(x_{3} x_{5}\right), k\right)
$$

But $x_{3} x_{5}$ is in the socle of $S$ and therefore we have as graded $S$-modules that $\left(x_{3} x_{5}\right) \stackrel{\sim}{\longrightarrow}$ $s^{-2} k$ so that the right summand of (1.10) is isomorphic to $E x t_{S}^{*-1, t}\left(s^{-2} k, k\right)$, i.e. to $\operatorname{Ext}_{S}^{*-1, t-2}(k, k)$. It remains to analyze the left summand of (1.10). But it is easy to see that $A n n_{S}\left(\left(x_{1}+x_{2}\right)\right)=I$ so that the graded sequence of $S$-modules

$$
0 \longrightarrow s^{-1} I \longrightarrow s^{-1} S \stackrel{\left(x_{1}+x_{2}\right)}{\longrightarrow} S
$$


where we multiply to the right with $x_{1}+x_{2}$ is exact. Therefore we have a short exact sequence of graded left $S$-modules:

$$
0 \longrightarrow s^{-1} I \longrightarrow s^{-1} S \longrightarrow\left(x_{1}+x_{2}\right) \longrightarrow 0
$$

leading to the isomorphism of left $\operatorname{Ext}_{S}^{*}(k, k)$-modules:

$$
\operatorname{Ext}_{S}^{*-1, t}\left(s^{-1} I, k\right) \stackrel{\sim}{\longrightarrow} \operatorname{Ext}_{S}^{*, t}\left(\left(x_{1}+x_{2}\right), k\right), \text { for } * \geq 1
$$

and using (1.9) once more we obtain that

$$
\operatorname{Ext}_{S}^{*-1, t}\left(s^{-1} I, k\right)=\operatorname{Ext}_{S}^{*-1, t-1}(I, k) \stackrel{\sim}{\longrightarrow} \operatorname{Ext}_{S}^{*, t-1}(S / I, k)
$$

leading to the final isomorphism (combining $(1.9),(1.10),(1.13),(1.14))$ :

$$
\operatorname{Ext}_{S}^{*, t}(S / I, k) \stackrel{\sim}{\longrightarrow} \operatorname{Ext}_{S}^{*-1, t-1}(S / I, k) \oplus \operatorname{Ext}_{S}^{*-1, t-2}(k, k)
$$

for $* \geq 1$, where the summand $\operatorname{Ext}_{S}^{*-1, t-2}(k, k)$ is non-zero only if $*=t-1$. This proves everything since we see, using (1.15) that $\operatorname{Ext}_{S}^{*, t}(S / I, k)$ needs a new $\operatorname{Ext}_{S}^{*}(k, k)$-generator for $*=t-1$ for each $t=2,3,4, \ldots$.

In particular we get if we introduce for any graded module $N$ over a graded $k$-algebra $G$ the double series

$$
P_{G}^{N}(x, y)=\sum_{i \geq 0, j \geq 0}\left|E x t_{G}^{i, j}(N, k)\right| x^{i} y^{j}
$$

(where as always for a $k$-vector space $V$ we denote by $|V|$ its dimension) and if we denote $P_{G}^{k}(x, y)$ by $P_{G}(x, y)$ we deduce from $(1.15)$ and the fact that $P_{S}(x, y)=1 /(1-2 x y)^{2}$ that

$$
P_{S}^{S / I}(x, y)=\frac{1}{1-x y}+\frac{x y^{2}}{(1-x y)(1-2 x y)^{2}}
$$

so that

$$
P_{S \propto s^{-1} S / I}(x, y)=\frac{P_{S}(x, y)}{1-x y P_{S}^{S / I}(x, y)}
$$

leading to the theorem:

THEOREM 1.1. The Orlik-Solomon algebra of the complex hyperplane arrangement $\mathcal{A}=\{x, y, z, x+y, x+z, y+z\}$ is the tensor product of the exterior algebra in one 
variable with an algebra $R$ whose Yoneda Ext-algebra $\operatorname{Ext}_{R}^{*}(k, k)$ has a bigraded generating series:

$$
P_{R}(x, y)=\frac{P_{S}(x, y)}{1-x y P_{S}^{M}(x, y)}=\frac{1-x y}{1-6 x y+12 x^{2} y^{2}-x^{2} y^{3}-8 x^{3} y^{3}}
$$

where $S$ and $M$ are defined above Furthermore, the Ext-algebra $\operatorname{Ext}_{R}^{*}(k, k)$ has global dimension 3 and it has 5 generators in degree 1 and needs one new generator in each degree $\geq 3$. In particular homotopy Lie algebra of $\mathcal{A}$ is not finitely generated.

We will come back to this result in the next section.

\section{The holonomy and homotopy Lie algebra of an arrangement.}

The preceding analysis of the $\mathcal{A}$ arrangement in section 1 was intended to give the "simplest possible proof" that the Ext-algebra $\operatorname{Ext}_{R}^{*}(k, k)$ was not finitely generated. However, in order to be able to analyze more cases we need a more general theory. I will here briefly describe the basics of such a theory and apply it as an alternative to our first case and then treat another case of arrangements where we can prove that the homotopy Lie algebra is also non-finitely presented. Note that the graded algebra $R$ of the previous section has Hilbert Series $1+5 z+7 z^{2}$. Let us now start with any algebra $R$ which is a quotient of an exterior algebra $E\left(x_{1}, x_{2}, \ldots, x_{n}\right)$ by a homogeneous ideal $J$ generated by elements of deegree $\geq 2$. Thus $R=E\left(x_{1}, \ldots, x_{n}\right) / J$. Let $m$ be the ideal of $R$ generated by $\left(x_{1}, \ldots, x_{n}\right)$, and consider the exact sequence of left $R$-modules:

$$
0 \longrightarrow m / m^{2} \longrightarrow R / m^{2} \longrightarrow R / m \longrightarrow 0,
$$

Now apply the functor $\operatorname{Ext}_{R}^{*}(, k)$ to the exact sequence (2.1). We get a long exact sequence which can be written as an exact sequence of left $\operatorname{Ext}_{R}^{*}(k, k)$-modules (we use the Yoneda product):

$$
0 \rightarrow s^{-1} \bar{S}_{m} \rightarrow s^{-1} \overline{\operatorname{Ext}}_{R}^{*}\left(R / m^{2}, k\right) \rightarrow \operatorname{Ext}_{R}^{*}(k, k) \otimes \operatorname{Ext}_{R}^{1}(k, k) \rightarrow \operatorname{Ext}_{R}^{*}(k, k) \rightarrow S_{m} \rightarrow 0
$$

where $S_{m}$ is defined as the image of the natural map

$$
\operatorname{Ext}_{R}^{*}(k, k) \longrightarrow \operatorname{Ext}_{R}^{*}\left(R / m^{2}, k\right)
$$

and where e.g. $\bar{S}_{m}$ means that we take the elements of $S_{m}$ of degrees $>0$ and where $s^{-1}$ is "the suspension" as before. Now the Ext-algebra $B=\operatorname{Ext}_{R}^{*}(k, k)$ is bigraded and we recall that its bigraded Hilbert series is denoted by

$$
P_{R}(x, y)=\sum_{i, j \geq 0}\left|\operatorname{Ext}_{R}^{i, j}(k, k)\right| x^{i} y^{j}=B(x, y)
$$


where as always for a $k$-vector space we denote by $|V|$ its dimension. The subalgebra $A$ of $\operatorname{Ext}_{R}^{*}(k, k)$ generated by $\operatorname{Ext}_{R}^{1}(k, k)$ is also bigraded but it is situated on the diagonal so that the corresponding bigraded Hilbert series

$$
A(x, y)=A(x y, 1) \stackrel{\text { def }}{=} A(x y)
$$

Now take the alternating sum of the two-variable Hilbert series of (2.2). We obtain:

$$
S_{m}(x, y)-B(x, y)+x y B(x, y)\left|m / m^{2}\right|-x \bar{P}_{R}^{R / m^{2}}(x, y)+x\left(S_{m}(x, y)-1\right)=0
$$

where

$$
\bar{P}_{R}^{R / m^{2}}(x, y)=\sum_{i>0, j \geq i}\left|E x t_{R}^{i, j}\left(R / m^{2}, k\right)\right| x^{i} y^{j}
$$

We now make three fundamental observations:

1) $A$ is a sub Hopf algebra of $B$ and therefore according to a result of Milnor and Moore [Mi-Mo] $B$ is free over $A$. Thus $S_{m}=B \otimes_{A} k$ has bigraded Hilbert series

$$
S_{m}(x, y)=B(x, y) / A(x y)
$$

2) If $m^{3}=0$ we have an isomorphism of left $\operatorname{Ext}_{R}^{*}(k, k)$-modules

$$
\overline{\operatorname{Ext}}_{R}^{*}\left(R / m^{2}, k\right) \simeq \operatorname{Ext}_{R}^{*}(k, k) \otimes \operatorname{Ext}_{R}^{1}\left(R / m^{2}, k\right)
$$

so that

$$
\bar{P}_{R}^{R / m^{2}}(x, y)=B(x, y) x y^{2}\left|m^{2} / m^{3}\right|
$$

Therefore the equality (14) can be written

$$
\frac{B(x, y)}{A(x y)}=B(x, y)-x y B(x, y)\left|m / m^{2}\right|+x^{2} y^{2} B(x, y)\left|m^{2} / m^{3}\right|-x\left(\frac{B(x, y)}{A(x y)}-1\right)
$$

which is another way of writing (divide by $x B(x, y)$ and use the notation $R(z)=1+$ $\left|m / m^{2}\right| z+\left|m^{2} / m^{3}\right| z^{2}$ for the Hilbert series of $R$ )

$$
1 / B(x, y)=(1+1 / x) / A(x y)-R(-x y) / x
$$


which is a formula due to Löfwall [Lö1].

3) In the case when $m^{3}=0$ the 3 middle terms of (2.2) are free $\operatorname{Ext}_{R}^{*}(k, k)$-modules so that $s^{-1} \bar{S}_{m}$ is a third syzygy of a minimal graded $\operatorname{Ext}_{R}^{*}(k, k)$-resolution of $S_{m}$. We therefore obtain the isomorphism:

$$
\operatorname{Tor}_{i, *}^{B}\left(k, S_{m}\right) \simeq \operatorname{Tor}_{i-3, *}^{B}\left(k, s^{-1} \bar{S}_{m}\right)=\operatorname{Tor}_{i-3, *-1}^{B}\left(k, \bar{S}_{m}\right) \quad \text { for } i \geq 3
$$

Now apply $\operatorname{Tor}_{i}^{B}(k$,$) to the exact sequence:$

$$
0 \longrightarrow \bar{S}_{m} \longrightarrow S_{m} \longrightarrow k \longrightarrow 0
$$

We get a long exact sequence:

$$
\ldots \rightarrow \operatorname{Tor}_{n+1}^{B}(k, k) \rightarrow \operatorname{Tor}_{n}^{B}\left(k, \bar{S}_{m}\right) \rightarrow \operatorname{Tor}_{n}^{B}\left(k, S_{m}\right) \stackrel{\varphi_{n}}{\rightarrow} \operatorname{Tor}_{n}^{B}(k, k) \rightarrow \operatorname{Tor}_{n-1}^{B}\left(k, \bar{S}_{m}\right) \ldots
$$

Furthermore, since $B$ is $A$-flat

$$
\operatorname{Tor}_{n}^{B}\left(k, S_{m}\right)=\operatorname{Tor}_{n}^{B}\left(k, B \otimes_{A} k\right)=\operatorname{Tor}_{n}^{A}(k, k)
$$

and $\varphi_{n}: \operatorname{Tor}_{n}^{A}(k, k) \rightarrow \operatorname{Tor}_{n}^{B}(k, k)$ is induced by the natural inclusion $A \rightarrow B$ which is split by a ring map in the other direction: Divide $B=\operatorname{Ext}_{R}^{*, *}(k, k)$ by the twosided ideal generated by $\oplus_{j>i>0} E x t_{R}^{i, j}(k, k)$. Thus the maps $\varphi_{n}$ in $(\mathrm{R})$ are monomorphisms and (2.13) splits into short exact sequences, using (2.11):

$$
0 \longrightarrow \operatorname{Tor}_{i, j}^{A}(k, k) \longrightarrow \operatorname{Tor}_{i, j}^{B}(k, k) \longrightarrow \operatorname{Tor}_{i+2, j+1}^{A}(k, k) \longrightarrow 0
$$

Now recall that for any graded algebra $A$ we have the following formula for the relation between its Hilbert series $A(z)$ and the Hilbert series $\operatorname{Tor}_{i, *}^{A}(k, k)(z)$ of the graded Tor: (cf. eg. Lemaire [Lem, Appendix A2]):

$$
\frac{1}{A(x)}=\sum_{i \geq 0}(-1)^{i} \operatorname{Tor}_{i, *}^{A}(k, k)(x)
$$

Now the formula (2.10) gives (replace $x$ by $x / y$ and put $y=0$ ) that $A(x)=(1-z) /(1-2 x)^{3}$ and now (2.15) gives since $\operatorname{gldim} A=3$ that $\operatorname{Tor}_{3, *}^{A}(k, k)(z)=z^{4} /(1-z)$ so that we see once more that $\operatorname{Tor}_{3, i}^{A}(k, k)$ is one-dimensional for all $i>3$. Note that $A$ is the enveloping algebra of a graded Lie algebra (the holonomy Lie algebra) whose ranks are equal to the ranks of the lower central series (LCS) of the fundamental group of the hyperplane complement. Now we can summarize: 
THEOREM 2.1. Let $R$ be a quotient of an exterior algebra (generators in degree 1 ) by a homogeneous ideal generated by elements of degree $\geq 2$. Let $m$ be the augmentation ideal of $R$. Assume that $m^{3}=0$. Let $B=\operatorname{Ext}_{R}^{*}(k, k)$ be the Yoneda Ext-algebra and let $A$ be the subalgebra of $B$, generated by $\operatorname{Ext}_{R}^{1}(k, k)$. Then the exact sequences (2.14) hold. In particular

a) $B$ is finitely generated if and only if the graded vector space $\operatorname{Tor}_{3, *}^{A}(k, k)$ has finite dimension.

b) $B$ is finitely presented if and only if the graded vector spaces $\operatorname{Tor}_{3, *}^{A}(k, k)$ and $\operatorname{Tor}_{4, *}^{A}(k, k)$ have finite dimension.

etc.

Remark 2.2. If not $m^{3}=0$ but more generally

$$
\overline{\operatorname{Ext}}_{R}^{*}\left(R / m^{i}, k\right) \longrightarrow \overline{\operatorname{Ext}}_{R}^{*}\left(R / m^{i+1}, k\right)
$$

is 0 for $i \geq 2$ then we have the same conclusion as in Theorem 2.1. Furthermore the formula $1 / P_{R}(x, y)=(1+1 / x) / A(x y)-R(-x y) / x$ is still valid under $(2.16)$ but here the Hilbert series $R(z)$ is a polynomial of degree $>2$.

Remark 2.3. In what follows we will denote $A$ by $R$ !

\section{Some other hyperplane arrangements}

Some of the cases from $[\mathrm{Su}]$ can be treated in the same way as in the end of the last part of section 2 . Here we just briefly describe the results for the so-called $X_{2}$-arrangement which is defined by the polynomial $x y z(x+y)(x-z)(y-z)(x+y-2 z)$. Now the analysis of the the Orlik-Solomon algebra leads as in section 2 to the analysis of the Yoneda Extalgebra of the quotient:

$$
R=\frac{E(a, b, c, d, e, f)}{(a b-a c+b c, a d, b e, c f, d e-d f+e f)}
$$

whose Hilbert series is $R(t)=1+6 t+10 t^{2}$. Furthermore, let

$$
S=\frac{E(a, b, c, d, e, f)}{(a b-a c+b c, a d, b e, c f)}
$$

and consider the ring map:

$$
S \longrightarrow S /(d e-d f+e f)=R
$$


It is not difficult to show that that (3.1) is a so-called Golod map (cf. [Le2] and the litterature cited there). One finds that $S^{!}(t)=(1-t)^{2} /(1-2 t)^{4}$, that $R^{!}(t)=(1-t)^{4} /(1-$ $2 t)^{5}$ and more precisely that $R^{!}$has global dimension 5 and that:

$$
\begin{aligned}
& \sum_{i \geq 0}\left|\operatorname{Tor}_{3, i}^{R^{!}}(k, k)\right| z^{i}=5 z^{4}+\frac{6 z^{5}}{(1-z)} \\
& \sum_{i \geq 0}\left|\operatorname{Tor}_{4, i}^{R^{!}}(k, k)\right| z^{i}=2 z^{6}+\frac{(6-z) z^{7}}{(1-z)^{2}}
\end{aligned}
$$

and

$$
\sum_{i \geq 0}\left|\operatorname{Tor}_{5, i}^{R^{!}}(k, k)\right| z^{i}=\frac{z^{10}}{(1-z)^{4}}
$$

Now using the theory from the end of section 2 we see that the homotopy Lie algebra of the arrangement $X_{2}$ is "extremely non-finitely presented": It needs an infinite number of generators (3.2), and the minimal number of relations between a minimal system of generators infinite (3.3) and the minimal number of relations between the relations is infinite (3.4).

We finish with two unsolved cases: Recall that the so-called non-Fano arrangement comes from the hyperplane arrangement defined by $x y z(x-y)(x-z)(y-z)(x+y-z)$ In this case the corresponding $R$ (we have eliminated one variable as above) has Hilbert series $(1+3 t)^{2}$ but the corresponding $R^{!}(t)$ is rather complicated. We have however managed to calculate the LCS-ranks two steps higher than in Suciu [Su], using the Backelin and al programme BERGMAN [B]; with the notations of [Su] we have $\phi_{8}=3148$ and $\phi_{9}=9857$, but for the last result we needed 64-bits $P S L$ on an AMD opteron machine with 12 GB of internal memory.

Another arrangement is the MacLane arrangement defined by $\left(\omega=e^{2 \pi i / 3}\right)$ the polynomial $x y z(y-x)(z-x)(z+\omega y)\left(z+\omega^{2} x+\omega y\right)\left(z-x-\omega^{2} y\right)$. Here we can still calculate many more LCS-ranks $\phi_{i}$ than in [Su] but unfortunately we do not see any general pattern: The $\phi_{i}$ for $i \geq 1$ are: $8,8,21,42,87,105,172,264,476,816,1516,2704,5068,9312,17484, \ldots$

\section{General remarks.}

In [De-Su] it is also asked if the enveloping algebra of the homotopy Lie algebra of an arrangement can have an irrational Hilbert series. In [Ro4] we have in particular described 
all homological possibilities for the quotient of an exterior algebra in $\leq 5$ variables by an ideal generated by $\leq 3$ quadratic forms (the ring-theoretical classification was obtained in $[\mathrm{E}-\mathrm{K}])$. It is only in 5 variables that we can obtain non-finitely generated Ext-algebras (only one case, just studied above) or Ext-algebras with an irrational Hilbert series (three cases). These three cases are as follows (the numbering of cases is from [Ro4]) (in all these three cases the Hilbert series $\left.R(t)=1+5 t+7 t^{2}\right)$ :

Case 12:

$$
R_{12}=\frac{E(x, y, z, u, v)}{(x y, x z+y u+z v, u v)} \quad \text { with } \quad \frac{1}{R_{12}^{!}(t)}=(1-2 t)^{2} \prod_{n=1}^{\infty}\left(1-t^{n}\right)
$$

Case 20:

$$
R_{20}=\frac{E(x, y, z, u, v)}{(y z+x u, y u+x v, z u+y v)} \quad \text { with } \quad \frac{1}{R_{20}^{!}(t)}=\prod_{n=1}^{\infty}\left(1-t^{2 n-1}\right)^{5}\left(1-t^{2 n}\right)^{3}
$$

Case 15:

$$
R_{15}=\frac{E(x, y, z, u, v)}{(y z+x u, x v, z u+y v)} \quad \text { with } \quad \frac{1}{R_{15}^{!}(t)}=(1-2 t) \prod_{n=1}^{\infty}\left(1-t^{2 n-1}\right)^{3}\left(1-t^{2 n}\right)^{2}
$$

But we can not see how any of these algebras could come up from some hyperplane arrangement. If we study quotients of $E(x, y, z, u, v)$ with four quadratic forms there are still three other quotients (this time with Hilbert series $R(t)=1+5 t+6 t^{2}$ ) which might have irrational $R^{!}(t)$ :

Case 21:

$$
\begin{aligned}
R_{21}= & \frac{E(x, y, z, u, v)}{(y z+x u, y u+x v, z u+y v, u v)} \text { with } R_{21}^{!}(t)=1+5 t+19 t^{2}+65 t^{3}+211 t^{4}+667 t^{5}+ \\
+ & 2081 t^{6}+6449 t^{7}+19919 t^{8}+61425 t^{9}+189273 t^{10}+583008 t^{11}+1795509 t^{12}+ \\
& +5529263 t^{13}+17026752 t^{14}+52431180 t^{15}+161452384 t^{16}+497162060 t^{17}+ \\
& +1530914456 t^{18}+4714152439 t^{19}+14516309322 t^{20}+44700127353 t^{21}+ \\
& +137645268696 t^{22}+423851580822 t^{23}+\ldots
\end{aligned}
$$

Case 22:

$$
\begin{aligned}
& R_{22}=\frac{E(x, y, z, u, v)}{(y z+x u, y u+x v, z u+y v, z v)} \quad \text { with } \quad R_{22}^{!}(t)=1+5 t+19 t^{2}+65 t^{3}+211 t^{4}+666 t^{5}+ \\
& +2071 t^{6}+6387 t^{7}+19609 t^{8}+60054 t^{9}+183672 t^{10}+561340 t^{11}+ \\
& +1714894 t^{12}+5237883 t^{13}+15996477 t^{14}+\ldots
\end{aligned}
$$


Case 33:

$$
\begin{aligned}
R_{33}= & \frac{E(x, y, t, u, v)}{(y z+x u, x v, z u+y v, u v)} \text { with } R_{33}^{!}(t)=1+5 t+19 t^{2}+65 t^{3}+212 t^{4}+675 t^{5}+2125 t^{6}+ \\
& +6653 t^{7}+\ldots(21 \text { terms })+483131948638003 t^{29}+1505474194810058 t^{30}+\ldots
\end{aligned}
$$

but the following formula gives in this last case an indication about theta functions :

$$
\begin{gathered}
\frac{1}{(1-t)^{2} R_{33}^{!}(t)}=1-3 t-t^{2}+t^{3}+2 t^{4}+3 t^{5}+t^{6}+ \\
+t^{7}-t^{8}-t^{9}-2 t^{10}-t^{11}-3 t^{12}-t^{13}-t^{14}-t^{15}+t^{17}+ \\
+t^{18}+2 t^{19}+t^{20}+t^{21}+3 t^{22}+t^{23}+t^{25}+t^{26}-t^{29}-t^{30} \ldots
\end{gathered}
$$

leading to the predictions that the coefficient for $t^{31}$ should be -2 and that more precisely the series should continue as $-2 t^{31}-t^{32}-t^{33}-t^{34}-3 t^{35}+\ldots$. But using the Backelin et al. programme BERGMAN [B] we have for the moment only been able to calculate the preceding series in degrees $\leq 30$ and no precise theory is in sight. But I still do not know if these last three cases come from some hyperplane arrangements. In higher embedding dimensions there are of course many more irrational series some of which could come from complex hyperplane arrangements ...

Remark 4.1. The case $R_{20}^{!}$(which comes from Jürgen Wisliceny and whose series was determined up to degree 67 by Czaba Schneider [Sch], Theorem 6.1 ) was completely determined in the super-Lie algebra case in [Lö-Ro] (where we had a periodicity 4). Here its treatment is easier (periodicity 2). Note that we have only described above what happens in characteristic 0 . In case 20 we have different $R_{20}^{!}(z)$ in all charcteristics and the same remark seems to be applicable to the cases $21,22,33$.

\section{Final remarks.}

It is interesting to note that 32-33 years ago Jean-Michel Lemaire was in his thesis [Lem] inspired by the Stallings group-theoretical example [St] (now used again in [Di-Pa-Su] !) to construct a finite simply-connected CW-complex $X$ such that the homology algebra of the loop space $H_{*}(\Omega X, \mathbf{Q})$ was not finitely presented (not even finitely generated). In [Ro1] I used a general recipee which in particular could be used to translate Lemaire's results to local commutative ring theory to obtain a local ring $(R, m)$ such that the Yoneda Extalgebra $\operatorname{Ext}_{R}^{*}(k, k)$ was not finitely generated, thereby solving in the negative a problem by Gerson Levin [Le1]. The example in section 2 above is just a skew-commutative variant of my example in [Ro1], but with a quick direct proof, which hopefully should satisfy mathematicians working with arrangements of hyperplanes. The theory of section 2 above 
can certainly be used for other hyperplane arrangements even when $m^{3} \neq 0$ (cf. Remark 2.2 and $[$ Ro3] $)$.

\section{REFERENCES}

[B] Backelin, Jörgen and al, BERGMAN, a programme for non-commutative Gröbner basis calculations available at http://servus.math.su.se/bergman/

[De-Su] Denham, Graham and Suciu, Alexander I, On the homotopy Lie algebra of an arrangement, Michigan Mathematical Journal 54, (2006), no 2, 319-340.

[Di-Pa-Su] Dimca, Alexandru, Papadima, Stefan and Suciu, Alexander I, Non-finitenes properties of fundamental groups of smooth projective varieties. Preprint available at arXiv:math.AG/0609456

[E-K] Eisenbud, David; Koh, Jee, Nets of alternating matrices and the linear syzygy conjectures. Adv. Math. 106 (1994), no. 1, 1-35.

[Lem] Lemaire, Jean-Michel, Algèbres connexes et homologie des espaces de lacets. (French) Lecture Notes in Mathematics, Vol. 422. Springer-Verlag, Berlin-New York, 1974. $x i v+134$ pp.

[Le1] Levin, Gerson, Two conjectures in the homology of local rings. J. Algebra 30 (1974), 56-74.

[Le2] Levin, Gerson Modules and Golod homomorphisms. J. Pure Appl. Algebra 38 (1985), no. 2-3, 299-304.

[Lö1] Löfwall, Clas On the subalgebra generated by the one-dimensional elements in the Yoneda Ext-algebra. Algebra, algebraic topology and their interactions (Stockholm, 1983), 291-338, Lecture Notes in Math., 1183, Springer, Berlin, 1986.

[Lö2] Löfwall, Clas, On the homotopy Lie algebra of a local ring. J. Pure Appl. Algebra 38 (1985), no. 2-3, 305-312

[Lö-Ro] Löfwall, Clas; Roos, Jan-Erik, A nonnilpotent 1-2-presented graded Hopf algebra whose Hilbert series converges in the unit circle. Adv. Math. 130 (1997), no. 2, $161-200$.

[Mi-Mo] Milnor, John W.; Moore, John C., On the structure of Hopf algebras. Ann. of Math. (2) 81 (1965) 211-264.

[Pa-Su] Papadima, Stefan and Suciu, Alexander I., When does the associated graded Lie algebra of an arrangement group decompose ?, Comment. Math. Helv. 81 (2006), 859-875.

[Ro1] Roos, Jan-Erik, Relations between Poincaré-Betti series of loop spaces and of local rings. Séminaire d'Algèbre Paul Dubreil 31ème année (Paris, 1977-1978), pp. 285322, Lecture Notes in Math., 740, Springer, Berlin, 1979. 
[Ro2] Roos, Jan-Erik, On the use of graded Lie algebras in the theory of local rings. Commutative algebra: Durham 1981 (Durham, 1981), pp. 204-230, London Math. Soc. Lecture Note Ser., 72, Cambridge Univ. Press, Cambridge-New York, 1982.

[Ro3] Roos, Jan-Erik, A computer-aided study of the graded Lie algebra of a local commutative Noetherian ring. J. Pure Appl. Algebra 91 (1994), no. 1-3, 255-315.

[Ro4] Roos, Jan-Erik, Homological properties of quotients of exterior algebras, in preparation; cf. Abstracts Amer.Math.Soc., 21 (2000), 50-51.

[Sch] Schneider, Czaba ,Computing Nilpotent Quotients in Finitely Presented Lie Rings. Discrete Mathematics \& Theoretical Computer Science. 1(1), pages 1-16, 1997.

[Sh-Yu] Shelton, Brad; Yuzvinsky, Sergey, Koszul algebras from graphs and hyperplane arrangements. J. London Math. Soc. (2) 56 (1997), no. 3, 477-490.

[St] Stallings, John ,A finitely presented group whose 3-dimensional integral homology is not finitely generated. Amer. J. Math. 85 (1963) 541-543.

[Su] Suciu, Alexander I. Fundamental groups of line arrangements: enumerative aspects. Advances in algebraic geometry motivated by physics (Lowell, MA, 2000), 43-79, Contemp. Math., 276, Amer. Math. Soc., Providence, RI, 2001. 